Original Article

\title{
An immunohistochemical study of the sciatic nerve in a rat knee immobilization model
}

\author{
Shinya Yoshida, $\mathrm{MS}^{1,2)^{*}}$, Taro Matsuzaki, $\mathrm{PhD}^{3)}$, Masahiro Hoso, $\mathrm{PhD}^{3)}$ \\ 1) Division of Health Sciences, Graduate School of Medical Science, Kanazawa University: 5-11-80 \\ Kodatsuno, Kanazawa, Ishikawa 920-0942, Japan \\ 2) Department of Rehabilitation, Kanazawa University Hospital, Japan \\ 3) Faculty of Health Sciences, Institute of Medical, Pharmaceutical and Health Sciences, Kanazawa \\ University, Japan
}

\begin{abstract}
Purpose] This study was performed to immunohistochemically evaluate changes in the periphery of the sciatic nerve in a rat model of knee immobilization, and to assess the effects of range of motion exercise. [Subjects and Methods] Twenty-one male rats were divided randomly into three groups: control (C), immobilized (I), and exercise (E group). Rats in the I and E groups had the right knee joint immobilized for 2 weeks. In the E group, range of motion exercise was also performed. After the experimental period, the periphery of the sciatic nerve was immunohistochemically observed. [Results] Immunohistochemical staining revealed that the myelin sheath and the perineurium in all groups were laminin positive. In the $\mathrm{C}$ and $\mathrm{E}$ groups, all rats showed normal staining. In contrast, 4 rats in the I group exhibited weak labeling. [Conclusion] Our results suggest that immobilization alters the perineurium at a molecular level and the range of motion exercise is essential for maintaining the environment of the perineurium.

Key words: Immunohistochemical staining, Perineurium, Laminin
\end{abstract}

(This article was submitted Dec. 2, 2015, and was accepted Dec. 18, 2015)

\section{INTRODUCTION}

Joint contracture occurs when joint movement and range of motion (ROM) are reduced, such as by immobilization of a joint with a plaster cast or an orthosis, or after prolonged bed rest. Contracture can be classified into three groups: arthrogenic, myogenic, and soft tissue ${ }^{1)}$. Decreased mobility and plasticity of the nervous system are also considered to cause ROM limitations; however, it remains unclear what changes occur in the nerves and periphery in the setting of contracture due to secondary disabilities or prolonged bed rest. We previously reported strong contact between nerve fiber bundles and the perineurium and decreased perineural spaces in immobilized rats ${ }^{2}$. Such contact could decrease nerve fiber bundle mobility and plasticity. There are several causes for this contact, including expression levels of cell adhesion molecule, physical factors such as perineurium contraction, and unknown factors. However, our previous hematoxylin-eosin (HE) results only revealed contact between nerve fiber bundles and the perineurium. Immunohistochemical studies are needed to clarify the detailed histopathologic changes in the periphery of the sciatic nerve in a model of rat knee immobilization.

Cell adhesion is roughly divided into cell-cell and cell-extracellular matrix (ECM) types ${ }^{3)}$. The former is adhesion between cells due to interactions between cell adhesion molecules, and the latter is based on interactions between cell adhesion molecules and the ECM. There are a number of cell adhesion factors. Laminins are a family of glycoproteins found predominantly in basement membranes. They are cross- or T-shaped heterotrimers consisting of one $\alpha$, one $\beta$, and one $\gamma$ chain ${ }^{4-6)}$. Basement membranes, axon surfaces, and Schwann cells are clearly labeled with polyclonal antibodies to laminin ${ }^{7}$.

Several animal studies have reported changes in muscles and joint components after joint immobilization. Sugama et al. ${ }^{8)}$

\footnotetext{
*Corresponding author. Shinya Yoshida (E-mail: yoshi511@med.kanazawa-u.ac.jp)

(C)2016 The Society of Physical Therapy Science. Published by IPEC Inc.

This is an open-access article distributed under the terms of the Creative Commons Attribution Non-Commercial No Derivatives (by-nc-nd) License $<$ http://creativecommons.org/licenses/by-nc-nd/4.0/>.
} 
demonstrated that salt-soluble collagen was significantly diminished after immobilization of the rat soleus muscle, and this result is suggestive of enhanced intermolecular cross-links corresponding to a stronger molecular structure. Hibino et al. ${ }^{9)}$ reported that insoluble collagen concentration significantly increased after immobilization, indicating an increase in collagen fibers with strong intermolecular cross-links contained in the muscle. Others have described increased synovial intima adhesion in the posterior capsule and hypothesized that this hyperplastic reaction was associated with infiltration into the articular cavity and adhesion to the articular cartilage surface ${ }^{10,11)}$. However, the mechanism of the strong contact between the nerve fiber bundles and the perineurium is unknown. The purpose of present study was to immunohistochemically evaluate changes in the periphery of the sciatic nerve in a rat model of knee immobilization and assess the effects of ROM exercise.

\section{SUBJECTS AND METHODS}

Twenty-one male Wistar rats (9 weeks old, 262-299 g) were randomly divided into three groups: control $(\mathrm{C}, \mathrm{n}=7)$, immobilized ( $\mathrm{I}, \mathrm{n}=7$ ), and exercise (E group, $\mathrm{n}=7$ ). The rats were not constrained in their cages and had free access to food and water. The animal room was kept at $22{ }^{\circ} \mathrm{C}$, and the animals were maintained under a 12-h light/dark cycle to suppress biological rhythm effects. This experiment was performed in accordance with the Guidelines for the Care and Use of Laboratory Animals of Kanazawa University, and the protocol was approved by the Animal Research Committee of Kanazawa University (Approval no. 132937).

Rats in the I and E groups had the right knee joint immobilized at $120^{\circ}$ flexion for 2 weeks with external fixation under deep anesthesia induced by an intraperitoneal injection of pentobarbital sodium ( $40 \mathrm{mg} / \mathrm{kg}$ ) (Fig. 1). The instruments for external fixation were a 0.8 -mm-diameter Kirschner wire, 4-mm diameter screw, and nuts. In the E group, ROM exercise was performed under anesthesia with isoflurane beginning the day after immobilization. Exercise time, exercise cycle, and pulling force were the same as previously described ${ }^{11)}$, and in this study, the right hindlimb was pulled $120^{\circ}$ ventrocranially to the longitudinal axis of the trunk. The right knee joints of E group rats were kept immobilized at all other times. The rats were able to freely move in their cages and had free access to food and water. At the end of the experimental period, the rats were sacrificed by an intraperitoneal injection of an overdose of pentobarbital sodium. Immediately after euthanasia, their right hindlimbs were disarticulated at the hip joint and fixed in 10\% neutral-buffered formalin for 72 hours. After fixation, the sciatic nerve was peeled off from the surrounding soft tissue and embedded in paraffin blocks that were cut into sections parallel to the longitudinal axis of the sciatic nerve at a thickness of $3 \mu \mathrm{m}$. The tissue sections were stained with HE for conventional histological examinations. For the immunohistochemical analysis, $3-\mu \mathrm{m}$ formalin-fixed and paraffin-embedded sections were prepared and labeled with the conventional avidin-biotin complex technique ${ }^{12}$. Sections were treated with proteinase $\mathrm{K}$ (S3020, Dako) for 5 minutes for antigen retrieval. Then, endogenous peroxidase was inactivated by the addition of hydrogen peroxidase for 20 minutes. Protein Block (X0909, Dako) was applied for 15 minutes to block nonspecific immunoglobulin binding. The sections were incubated with polyclonal rabbit anti-laminin (Z0097, Dako, dilution 1:100) overnight at room temperature and then incubated with horseradish peroxidase-conjugated to goat anti-rabbit immunoglobulin (K4003, Dako). Antibody binding was visualized using the Liquid DAB Substrate Chromogen System (K3468, Dako) for 60 seconds at room temperature. All sections were counterstained with hematoxylin.

We examined the periphery of the sciatic nerve under an optical microscope (BX51, Olympus). Three investigators independently confirmed the histopathologic findings. We classified immunohistochemical staining into three categories in accordance with the degree of positive laminin staining in the adhesion areas between the nerve fiber bundles and the perineurium (Fig. 2).

\section{RESULTS}

Immunohistochemical staining revealed that the myelin sheath and innermost layer of the perineurium in all groups were laminin positive. In the $\mathrm{C}$ and $\mathrm{E}$ groups, all rats showed normal staining. In contrast, samples from 4 of the 7 rats in the $\mathrm{I}$ group exhibited weak staining (Table 1).

\section{DISCUSSION}

In the present study, the immunohistochemical changes in the periphery of the sciatic nerve of rats that underwent knee immobilization were evaluated, as well as the effects of ROM exercise on them. Magari ${ }^{13)}$ and Thomas ${ }^{14)}$ reported that only the perineurium possesses basement membranes while the endoneurium and epineurium were collagenous connective tissue layers or mere connective tissue. Kuechere and colleagues reported that laminin is produced by Schwann cells and is present on the surfaces of axons, basement membranes, and Schwann cells ${ }^{15}$. In this study, these regions of the rats sciatic nerves showed similar staining for laminin, and our results show that the innermost layer of the perineurium in all of the groups was stained laminin-positive. However, the staining was weaker in the perineurium of the I group than that of the myelin sheath. These results suggest that immobility by joint fixation creates different conditions in the perineurium compared to the normal situation, and that ROM exercise helped to maintain the basic environment of the perineurium in the E group. The essential functions of laminin are roughly divided into two: interactions with other ECM proteins involved in architectural 


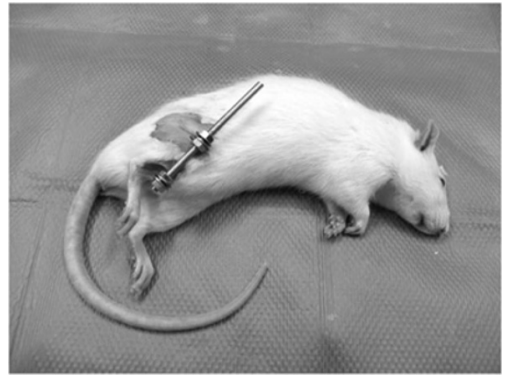

Fig. 1. Immobilization model with external fixation

Table 1. Classification results of the immunohistochemical staining

\begin{tabular}{lccc}
\hline & Normal & Weak & Unclassified \\
\hline C group & 7 & 0 & 0 \\
I group & 1 & 4 & 2 \\
E group & 7 & 0 & 0 \\
\hline
\end{tabular}

C group: control group; I group: immobilization group; E group: immobilization + ROM exercise group

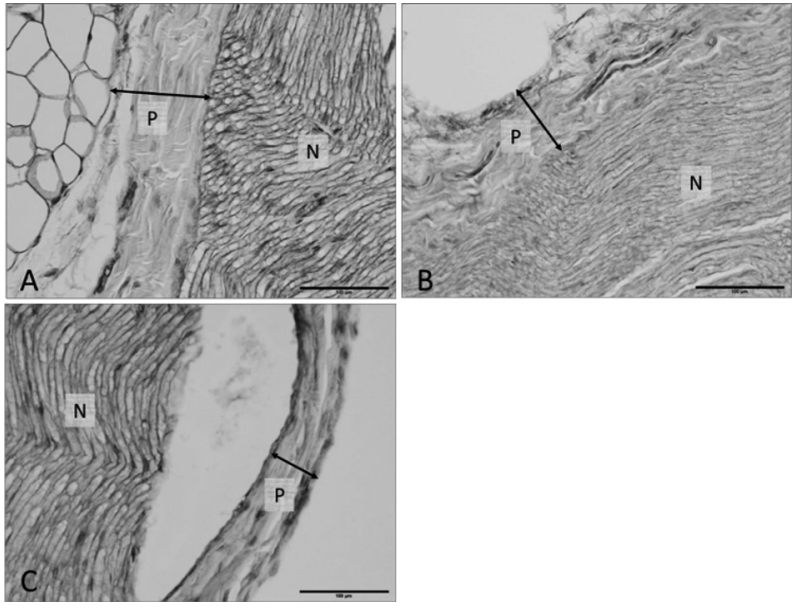

Fig. 2. Original classification of immunohistochemical staining (Scale bar: $100 \mu \mathrm{m}$ )

A: Normal type, strongly laminin positive at the adhesion sites; B: Weak type, partial weak staining at the adhesion sites; C: Unclassified, no adhesion sites

P: Perineurium, N: the bundle of nerve fibers

function, such as assembly and stability within the basement membrane; and interactions with cell surface receptors involved in adhesion, migration, and differentiation ${ }^{4}$. We hypothesize that joint immobilization or ROM exercise affects these laminin functions, but the detailed mechanism remains unclear.

The results of this study possibly provide evidence of the need to determine the appropriate and effective ROM exercise in the field of physiotherapy and rehabilitation.

This study had some limitations. First only the laminin expression was assessed; therefore, further studies that examine the expression of other cell adhesion factors are needed to clarify the detailed pathologic condition in the periphery of the sciatic nerve in the immobilized rat knee joint. Also, this study did not include a quantitative evaluation.

In conclusion, our results suggest that immobilization alters the perineurium at a molecular level. This study is the first attempt to immunohistochemically examine changes in the periphery of the sciatic nerve in a rat model of knee immobilization.

\section{REFERENCES}

1) Halar EM, Bell KR: Rehabilitation Medicine Principles and Practice, 3rd ed. Philadelphia: JB Lippincott-Raven, 1988, pp 1015-1034.

2) Yoshida S, Matsuzaki T, Kamijo A, et al.: Histopathological changes in the periphery of the sciatic nerve of rats after knee joint immobilization. J Phys Ther Sci, 2013, 25: 623-626. [Medline] [CrossRef]

3) Gumbiner BM: Cell adhesion: the molecular basis of tissue architecture and morphogenesis. Cell, 1996, 84: 345-357. [Medline] [CrossRef]

4) Aumailley M: The laminin family. Cell Adhes Migr, 2013, 7: 48-55. [Medline] [CrossRef]

5) Durbeej M: Laminins. Cell Tissue Res, 2010, 339: 259-268. [Medline] [CrossRef]

6) Alberts B, Alexander J, Julian L, et al.: Molecular biology of the cell, 6th ed. New York: Garland Science, 2014, pp 1069-1073.

7) Patton BL, Miner JH, Chiu AY, et al.: Distribution and function of laminins in the neuromuscular system of developing, adult, and mutant mice. J Cell Biol, 1997, 139: 1507-1521. [Medline] [CrossRef]

8) Sugama S, Tachino K, Haida N: Effect of immobilization on solubility of soleus and gastrocnemius muscle collagen: biochemical studies on collagen from soleus and gastrocnemius muscles of rat. J Jpn Phys Ther Assoc, 1999, 2: 25-29. [Medline] [CrossRef]

9) Hibino I, Okita M, Inoue T, et al.: Effect of immobilization on insoluble collagen concentration and type I and type III collagen isoforms of rat soleus muscle. J Jpn Phys Ther Assoc, 2008, 11: 1-6. [Medline] [CrossRef] 
10) Trudel G, Seki M, Uhthoff HK: Synovial adhesions are more important than pannus proliferation in the pathogenesis of knee joint contracture after immobilization: an experimental investigation in the rat. J Rheumatol, 2000, 27: 351-357. [Medline]

11) Matsuzaki T, Yoshida S, Kojima S, et al.: Influence of ROM exercise on the joint components during immobilization. J Phys Ther Sci, 2013, 25: 1547-1551. [Medline] [CrossRef]

12) Hsu SM, Raine L, Fanger H: The use of antiavidin antibody and avidin-biotin-peroxidase complex in immunoperoxidase technics. Am J Clin Pathol, 1981, 75: 816-821. [Medline]

13) Magari S: The spinal cord and the lymphatic system. in Lymphangiology. edited by Foldi M et al. F.K. Schattauer Verlag. Stuttgart, 1983, pp 509-533.

14) Thomas PK: The connective tissue of peripheral nerve: an electron microscope study. J Anat, 1963, 97: 35-44. [Medline]

15) Kuecherer-Ehret A, Graeber MB, Edgar D, et al.: Immunoelectron microscopic localization of laminin in normal and regenerating mouse sciatic nerve. J Neurocytol, 1990, 19: 101-109. [Medline] [CrossRef] 